\title{
Considerações sobre Coprophanaeus ensifer (Germar) (Coleoptera, Scarabaeidae) em um remanescente de Mata Atlântica no Estado da Paraíba, Brasil
}

\author{
Ana Aline Endres ${ }^{1}$, Malva Isabel Medina Hernández² \& Antônio José Creão-Duarte ${ }^{3}$
}

1Programa de Pós Graduação em Ciências Biológicas-Zoologia, DSE, CCEN, UFPB. alinendres@hotmail.com
${ }^{2,3}$ Departamento de Sistemática e Ecologia, CCEN, Universidade Federal da Paraíba, 58051-900 João Pessoa-PB, Brasil.
malvamh@yahoo.com, creao@dse.ufpb.br

\begin{abstract}
Notes about Coprophanaeus ensifer (Germar) (Coleoptera, Scarabaeidae) in a remnant of the Atlantic Forest in the State of Paraíba, Brazil. Insects of the family Scarabaeidae feed on organic matter in decomposition, participating actively in biogeocycling of nutrients. C. ensifer is a large-sized necrophagous beette that occurs in tropical forests. In the present work we aimed to record some ecological characteristics of this species regarding the seasonality and bait attractivity. Thirteen samples were performed between December/1998 and December/1999 in the Mata do Buraquinho, a remnant of the Atlantic Forest in João Pessoa, PB. Seventy-one specimens were captured in four baited pitfall traps: 35 on pork meat, 22 on kidney, and 14 on bovine meat. There was no significant difference among the baits with respect to their stimuli for attracting the insects. No specimens of $C$. ensifer were trapped on baits of liver. The monthly abundance of insects is positively correlated the precipitation $\left(r_{s}=0,65 ; \mathrm{p}<0,05\right)$ and humidity $\left(r_{s}=0,55 ; \mathrm{p}<0,05\right)$ and inversely with temperature $\left(r_{s}=-0,70 ; \mathrm{p}<0,01\right)$. The specimens were collected only from April to September, within the rainy period, an aspect that corroborates the seasonal pattern of occurrence.
\end{abstract}

KEYWORDS. Abundance, attractivity of baits, ecology, seasonality.

RESUMO. Considerações sobre Coprophanaeus ensifer (Germar) (Coleoptera, Scarabaeidae) em um remanescente de Mata Atlântica no estado da Paraíba, Brasil. Os insetos da família Scarabaeidae se alimentam da matéria orgânica em decomposição, participando ativamente da ciclagem de nutrientes. C. ensifer é um necrófago de grande porte que ocorre em áreas de florestas tropicais. Este estudo teve por objetivo conhecer o padrão sazonal da espécie neste ambiente e avaliar a atratividade das iscas utilizadas. Foram realizadas 13 coletas entre os meses de Dezembro/98 e Dezembro/99 na Mata do Buraquinho, um remanescente de Mata Atlântica em João Pessoa, PB. Foram coletados 71 espécimes usando armadilhas com quatro tipos de isca: $35 \mathrm{em}$ carne de porco, $22 \mathrm{em}$ rim bovino e 14 indivíduos em carne bovina, não havendo diferenças significativas entre as iscas em relação a sua atratividade para com os insetos. Não foi coletado nenhum espécime em fígado bovino. A abundância mensal esteve diretamente correlacionada com a precipitação $(r=0,65$; $\mathrm{p}<0,05)$ e com a umidade $\left(r_{s}=0,55 ; \mathrm{p}<0,05\right)$ e inversamente com a temperatura $\left(r_{s}=-0,70 ; \mathrm{p}<0,01\right)$. Os espécimes somente foram coletados entre os meses de Abril a Setembro, coincidindo com o período chuvoso na região, o que corrobora o padrão sazonal da espécie.

PALAVRAS-CHAVE. Abundância, atratividade de iscas, ecologia, sazonalidade.

A família Scarabaeidae tem cerca de cerca de 5000 espécies e grande abundância de indivíduos (Hanski 1991). A maioria das espécies está restrita a áreas onde a precipitação ultrapassa $250 \mathrm{~mm}$ por ano e não apresentam temperaturas abaixo de $15^{\circ} \mathrm{C}$. A sazonalidade de suas populações é nítida em florestas tropicais úmidas, onde a abundância de indivíduos se eleva após o início das chuvas (Halffter \& Matthews 1966; Janzen 1983; Stumpf 1986).

Adultos e larvas da família Scarabaeidae alimentam-se principalmente de carniça (necrófagos) e fezes (coprófagos), sendo agentes importantes na degradação de matéria orgânica no solo. Este hábito alimentar permite estudos populacionais através de capturas por armadilhas que utilizam iscas para sua atração. Acredita-se que várias espécies desta família invadiram as florestas da América do Sul e passaram da coprofagia para a necrofagia devido, provavelmente, às extinções dos grandes mamíferos durante o Pleistoceno, criando novos nichos e diminuindo a competição (Cambefort 1991).

O gênero Coprophanaeus d'Olsoufieff, 1924 (Subfamília Onitinae) inclui em sua maioria espécies necrófagas, sendo encontradas geralmente em cadáveres frescos nos períodos crepusculares a noturnos, voando sobre o alimento por curtos períodos de tempo, pousando justamente sobre o recurso alimentar (Halffter \& Mathews 1966; Halffter \& Edmonds 1982; Otronen 1988; Gill 1991). A forma de nidificação destas espécies consiste na construção de bolas a partir do recurso alimentar, que servem de abrigo e alimento às larvas, aumentando desta maneira as chances de sucesso reprodutivo (Teicher 1959; Halffter et al. 1974; Halffter \& Edmonds 1982). A construção de ninhos associada à baixa fecundidade das fêmeas e à 
redução da competição demonstram que estas espécies podem ser consideradas estrategistas-k (Halffter \& Halffter 1989; Cambefort \& Hansky 1991).

A espécie Coprophanaeus (Megaphanaeus) ensifer (Germar, 1824) está incluída entre as maiores espécies da região neotropical, com cerca de $50 \mathrm{~mm}$, sendo preferencialmente necrófaga (Gill 1991). Apresenta um corno no pronoto de tamanho similar em machos e fêmeas (Otronen 1988). É encontrada somente na América do Sul (Halffter \& Edmonds 1982; Otronen 1988), apresentando uma ampla distribuição dentro do continente, com registros para o Paraguai, Argentina e Brasil sendo desconhecida sua exata distribuição no Brasil, mas sabe-se que é freqüente em algumas regiões de São Paulo (Pessoa \& Lane 1941; Otronen 1988) e do Mato Grosso do Sul (Flechtmann et al. 1995).

Este trabalho teve como objetivo avaliar a atratividade de iscas para C. ensifer e conhecer o padrão sazonal da espécie em um remanescente de Mata Atlântica do Nordeste brasileiro.

O estudo foi realizado na Mata do Buraquinho, atual Jardim Botânico Benjamim Maranhão, um fragmento de Mata Atlântica de 517 ha, localizada no perímetro urbano do Município de João Pessoa - PB ( $7^{\circ} 10^{\prime}$ S; $\left.35^{\circ} 50^{\prime} \mathrm{W}\right)$. A área caracteriza-se por sua formação densa, sempre verde, com clima quente e úmido, temperatura média anual em torno de $25^{\circ} \mathrm{C}$, umidade relativa do ar de $80 \%$ e índices pluviométricos de $1600 \mathrm{~mm}$ anuais em média, com grandes variações de ano para ano (Lima \& Heckendorff 1985).

Foram utilizadas quatro armadilhas de queda tipo "pitfall", modificadas de Lobo et al. (1988), compostas de um tubo de PVC enterrado no solo com uma estrutura central para depositar a isca a $30 \mathrm{~cm}$ de profundidade. As armadilhas foram distribuídas ao longo de uma linha, espaçadas $20 \mathrm{~m}$ uma da outra e iscadas respectivamente com $100 \mathrm{~g}$ de carne suína, 100 $\mathrm{g}$ de carne bovina, $100 \mathrm{~g}$ de rim bovino e $100 \mathrm{~g}$ de fígado bovino frescos. As coletas se estenderam por sete dias a cada mês, de dezembro de 1998 a dezembro de 1999, sendo os insetos recolhidos das armadilhas a cada $24 \mathrm{~h}$. A identificação dos insetos foi confirmada pelo taxonomista Fernando Z. Vaz-deMello, e toda a série está incorporada no acervo da Coleção Entomológica do Departamento de Sistemática e Ecologia da Universidade Federal da Paraíba, João Pessoa-PB.

Os dados meteorológicos mensais de temperatura média, precipitação pluvial e umidade relativa do ar do município de João Pessoa foram obtidos com o INMET-PE.

As relações entre o número de indivíduos e as variáveis ambientais foram mensuradas utilizando o Método de Correlação de Spearman. Para avaliar diferenças entre o poder de atração das iscas, foi aplicado o teste Kruskal-Wallis (Statsoft 1988).

Durante o período de coleta foram capturados 71 indivíduos de C. ensifer, $35 \mathrm{em}$ carne de porco, $22 \mathrm{em}$ rim, $14 \mathrm{em}$ carne bovina e nenhum em fígado, ficando este último excluído das análises. Mesmo com cerca de $50 \%$ do total de insetos coletados em armadilhas iscadas com carne de porco, não houve diferença significativa entre o número de indivíduos e o poder de atração das três iscas $(H=1,27 ; \mathrm{p}=0,5)$.

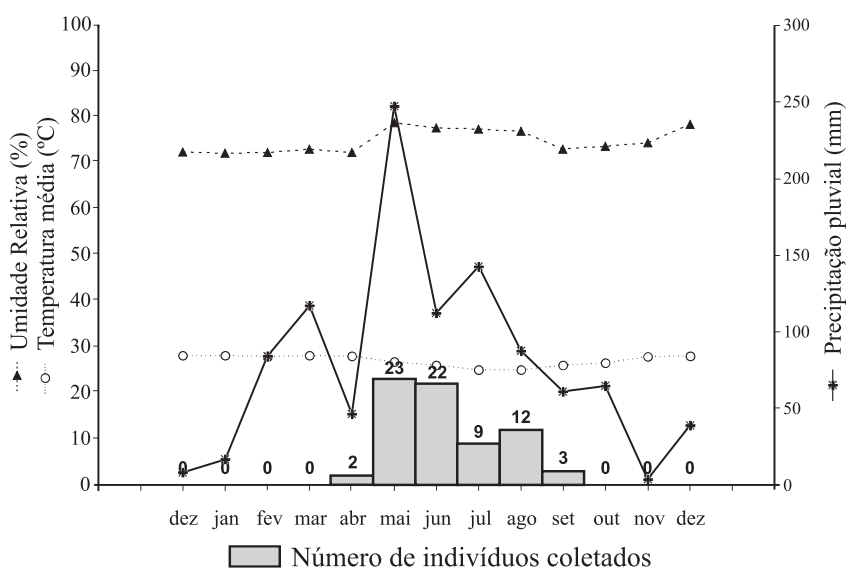

Fig. 1. Abundância de Coprophanaeus ensifer, temperatura média $\left({ }^{\circ} \mathrm{C}\right)$, umidade relativa média (\%) e precipitação pluvial mensal (mm) registradas durante os meses de coleta (Dez/98 a Dez/99) na Mata do Buraquinho-PB.

A presença da espécie foi registrada somente de abril a setembro (Figura 1). A abundância esteve positivamente correlacionada à precipitação $\left(r_{s}=0,65 ; \mathrm{p}<0,05\right)$ e à umidade relativa $\left(r_{s}=0,55 ; \mathrm{p}<0,05\right)$ e inversamente correlacionada com a temperatura $\left(r_{s}=-0,70 ; \mathrm{p}<0,01\right)$. Este padrão se explica já que a temperatura no Nordeste brasileiro, além de não apresentar fortes variações, diminui durante o período chuvoso, de forma que os insetos aparecem nos períodos de alta precipitação e umidade, o que coincide com a época de temperaturas mais amenas na região (Figura 1).

A ocorrência de C. ensifer no Nordeste do Brasil foi registrada pela primeira por Endres (2003). Esta espécie encontra-se distribuída principalmente nos remanescentes de Mata Atlântica da Paraíba, tendo sido coletada também na Reserva Biológica Guaribas, localizada ao norte do Estado, tanto em áreas de Mata como em áreas adjacentes cobertas por vegetação característica de Tabuleiro Nordestino, semelhante ao Cerrado do Brasil Central (Endres 2003). Esta espécie não foi coletada na Caatinga, embora 20 outras espécies de Scarabaeidae tenham sido coletadas (Hernández, 2005), portanto sua distribuição deve estar restrita aos locais de Mata e suas proximidades, já que os baixos níveis de precipitação em outras regiões do Estado, aparentemente representam um fator limitante para o seu estabelecimento.

Podemos constatar o hábito necrófago da espécie, como descrito na literatura (Halffter \& Mathews 1966; Halffter \& Edmonds 1982; Gill 1991; Otronen 1988) e fortemente generalista. $\mathrm{O}$ fato da espécie não ter sido encontrada em armadilhas iscadas com fígado sugere uma falta de atratividade desta isca.

A sazonalidade observada em C. ensifer reflete um padrão comum entre as espécies de Scarabaeidae coletadas em diversos tipos de vegetação, onde o aumento da precipitação pluvial faz emergir os adultos (Hilje 1996; Hill 1993; Davis 1987; Stumpf 1986; Louzada \& Lopes 1997). Durante o período seco do ano (normalmente de setembro a fevereiro na região) não 
houve captura de adultos da espécie, o que sugere que o grande porte dos indivíduos implique em uma característica limitante em relação aos fatores climáticos, reduzindo a atividade de adultos durante o período seco. A manutenção do equilíbrio hídrico pelo inseto deve ser possível quando a pluviosidade atinge um nível que permita a manutenção da água do corpo, já que a perda por evaporação, produto das altas temperaturas, deve ser alta. Esta idéia é reforçada pelo comportamento crepuscular e noturno da espécie (Halffter \& Matthews 1966; Gill, 1991), fazendo com que o período de atividade seja nos horários mais amenos do dia.

Agradecimentos. A Fernando Z. Vaz-de-Mello pela identificação da espécie; à diretoria do Jardim Botânico Benjamim Maranhão por autorizar as coletas; ao INMET por fornecer os dados meteorológicos e ao Dr. Alexandre Vasconcellos pelos comentários do manuscrito. M.I.M. Hernández agradece ao CNPq pelo suporte financeiro (bolsa DCR, Proc: No 301303/01-0).

\section{REFERÊNCIAS}

Cambefort, Y. 1991. From saprophagy to coprophagy, p. 22-35. In: I. Hanski \& Y. Cambefort (eds.). Dung Beetle Ecology. Princeton University Press, Princeton. 481 p.

Cambefort, Y. \& I. Hanski. 1991. Dung Beetle Population Biology, p. 36-50. In: I. Hanski \& Y. Cambefort (eds.). Dung Beetle Ecology. Princeton University Press, Princeton. 481 p.

Davis, A. L. V. 1987. Geographical distribuition of dung beetles (Coleoptera: Scarabaeidae) and their seasonal activity in southwestern Cape Province. Journal of Entomology Society of South Africa 50: 275-285.

Endres, A. A. 2003. Diversidade e Análise Faunística de Scarabaeidae (Coleoptera) em áreas de Mata e de Tabuleiro na Reserva Biológica Guaribas, Mamanguape-Pb. Dissertação de Mestrado, UFPB. 46 p.

Flechtmann, C. A. H.; S. R. Rodrigues \& M. C. Z. Seno. 1995. Controle biológico da mosca-dos-chifres (Haematobia irritans irritans) em Selvíria, Mato Grosso do Sul. 3. Levantamento de espécies fimícolas associadas à mosca. Revista Brasileira de Entomologia 39: 249-258.

Gill, B. D. 1991. Dung Beetles in American Tropical Forest, p. 211229. In: I. Hanski \& Y. Cambefort (eds.). Dung Beetle Ecology. Princeton University Press, Princeton. $481 \mathrm{p}$.

Halffter, G. \& E. G. Matthews. 1966. The natural history of dung beetles of the subfamily Scarabaeinae (Coleoptera: Scarabaeidae). Folia Entomológica Mexicana (12/14): 1-312.
Halffter, G \& W. D. Edmonds. 1982. The Nesting Behavior of Dung Beetles (Scarabaeinae). An Ecological and Evolutive Approach. Man and the Biosphere Program UNESCO. México D.F. 177 p.

Halffter, G. \& V. Halffter. 1989. Behavioral evolution of the nonrolling roller beetles. Acta Zoológica Mexicana 32: 3-53.

Halffter, G.; V. Halffter \& G. I. López. 1974. Phanaeus behaviour: food transportation and bisexual cooperation. Environmental Entomology 3: 341-345.

Hanski, Y. 1991. The dung insect community, p. 5-21. In: I. Hanski \& Y. Cambefort (eds.). Dung Beetle Ecology. Princeton University Press, Princeton. $481 \mathrm{p}$.

Hernández, M. I. M. 2005. Artrópodes: Besouros Scarabaeidae (Coleoptera) do Curimataú, Paraíba, Brasil, p. 369-380. In: F. S. Araújo, M. J. N. Rodal \& M. R. V. Barbosa (eds.). Análise das Variações da Biodiversidade do Bioma Caatinga. Suporte a Estratégias Regionais de Conservação. Ministério do Meio Ambiente. 434 p.

Hilje, L. 1996. Estacionalidad de adultos de Scarabaeidae (Coleoptera) en Barva, Costa Rica. Revista Biológica Tropical 44: 719-729.

Hill, C. J. 1993. The species composition and seasonality of an assemblage of tropical Australian dung beetles (Coleoptera: Scarabaeidae: Scarabaeinae). Australian Entomologist 20: 121126.

Jansen, D. H. 1983. Seasonal change in abundance of large nocturnal dung beetles (Sacarabaeidae) in Costa Rican deciduous forest and adjacent horse pasture. Oikos 41: 274-283.

Lima, P. J. \& W. D. Heckendorff. 1985. Climatologia, p 34-44. In: Governo do Estado da Paraíba, Secretaria da Educação, Universidade Federal da Paraíba (eds.). Atlas Geográfico do Estado da Paraíba. João Pessoa, Grafset.

Lobo, J. M.; F. Martín-Piera \& C. M. Veiga. 1988. Las trampas pitfall con cebo, sus posibilidades en el estudio de las comunidades coprófagas de Scarabaeoidea (Col); I: Características determinantes de su capacidad de captura. Revue d'Ecologie et Biologie du Sol 25: $77-100$.

Louzada, J. N. C. \& F. S. Lopes. 1997. A comunidade de Scarabaeidae copro-necrófagos (Coleoptera) de fragmento de Mata Atlântica. Revista Brasileira de Entomologia 41: 117-121.

Otronen, M. 1988. Intra- and intersexual interactions at breeding burrows in the horned beetle, Coprophanaeus ensifer. Animal Behaviour 36: 741-748.

Pessoa, S. B \& F. Lane. 1941. Coleópteros necrófagos de interesse médico-legal. Arquivo de Zoologia do Estado de São Paulo 2: 389-504.

Statsoft, Inc. 1998. STATISTICA for Windows. Tulsa, OK.

Stumpf, I. V. K., 1986. Study of the scarab fauna in Mandirituba, Parana, Brazil. Acta Biológica Paranaense 15: 125-153.

Teicher, M. 1959. Die bodenbiologische Bedeutung der coprophagen Lamellicornier. Wissenschafliche Zeitschrift der Martin Luther-Universität Halle-Wittenberg 8: 879-882. 